\title{
Face Recognition Algorithm Based on Adjacent Pixel Intensity Difference Quantization in Rectangular Coordinate Plane
}

\author{
Feifei Lee, Koji Kotani, Qiu Chen, and Tadahiro Ohmi
}

\begin{abstract}
We have proposed a very simple yet highly reliable face recognition algorithm using Adjacent Pixel Intensity Difference Quantization (APIDQ) histogram previously. In this paper, we present a modified quantization method to improve recognition performance. After the intensity variation vectors for all the pixels in an image are calculated, each vector is quantized directly in $(d \mathrm{Ix}, d \mathrm{Iy})$ plane instead of $r-\theta$ plane. By counting the number of elements in each quantized area in the $(d \mathrm{Ix}, d \mathrm{Iy})$ plane, a histogram can be created. This histogram, obtained by APIDQ for facial images, is utilized as a very effective personal feature. Furthermore, by utilizing rough location information of facial parts, the facial area is divided into 5 individual parts, and then APIDQ is applied on each facial component. Recognition results are firstly obtained from different parts separately and then combined by weighted averaging. Experimental results show that the top of recognition rate of the whole research groups is achieved by using FB task of the publicly available face database of FERET.
\end{abstract}

Index Terms-Face recognition, adjacent pixel intensity difference quantization (APIDQ), histogram.

\section{INTRODUCTION}

As a more natural and effective person identification method compared with that utilizing other biometric features such as voice, fingerprint, iris pattern, etc., face recognition has received significant attention in the last two decades due to requirement of security and law enforcement applications [1]. A lot of face recognition algorithms have been proposed [2-12]. These algorithms can be roughly divided into two main categories, namely, feature-based and appearance-based.

In the feature-based approaches [4], [5], recognition is based on the relationship between human facial features such as eye, mouth, nose, profile silhouettes and face boundary. Appearance-based approaches [6]-[9] attempt to capture and define the face as a whole. The face is treated as a two dimensional pattern of intensity variation. Under this approach, the face is matched through finding its underlying statistical regularities. Principal component analysis (PCA) is a typical appearance-based technique [6], which casts about for a set of projection vectors projecting the facial image data into a subspace based on the variation in energy. Fisherface is

Manuscript received June 12, 2012; revised July 6, 2012

Feifei Lee, Qiu Chen, and Tadahiro Ohmi are with New Industry Creation Hatchery Center, Tohoku University, Sendai, 980-8579 Japan (e-mail: fei@fff.niche.tohoku.ac.jp).

Koji Kotani is with Department of Electronics, Graduate School of Engineering, Tohoku University, Sendai, 980-8579 Japan. another representative in this category, which incorporates linear discriminant analysis (LDA) to extract the most discriminant features and to reduce the dimensionality [7]. However, these techniques are highly complicated and are computationally power hungry, making it difficult to implement them into real-time face recognition applications.

Previously, we have developed a very simple, yet highly reliable face recognition method called Adjacent Pixel Intensity Difference Quantization (APIDQ) Histogram Method, which achieved the real-time face recognition [13]. At each pixel location in an input image, a 2-D vector (composed of the horizontally adjacent pixel intensity difference $(d \mathrm{Ix})$ and the vertically adjacent difference $(d \mathrm{Iy})$ ) contains information about the intensity variation angle $(\theta)$ and its amount $(r)$. After the intensity variation vectors for all the pixels in an image are calculated and plotted in the $r-\theta$ plane, each vector is quantized in terms of its $\theta$ and $r$ values. By counting the number of elements in each quantized area in the $r$ - $\theta$ plane, a histogram can be created. This histogram, obtained by APIDQ for facial images, is utilized as a very effective personal feature. By combining APIDQ with an appropriate low pass filter as pre-treatment of a facial image, the useful features for face recognition can be extracted. Experimental results show a recognition rate of $95.7 \%$ for 400 images of 40 persons (10 images per person) from the publicly available AT\&T face database. In this paper, we focus on the quantization method of APIDQ. We found that quantization directly in $(d \mathrm{Ix}, d \mathrm{Iy})$ plane is more efficient in computation and can get higher recognition rate instead of $r$ - $\theta$ plane.

In section II, we introduce proposed modified Adjacent Pixel Intensity Difference Quantization (APIDQ) histogram method. Experimental results will be discussed in section III. Finally, conclusions are given in section IV.

\section{PROPOSED METHOD}

Fig. 1 shows the processing steps of proposed modified Adjacent Pixel Intensity Difference Quantization (APIDQ) histogram method. Low-pass filtering is first carried out before APIDQ using a simple 2-D moving average filter. This low-pass filtering is essential for reducing the high-frequency noise and extracting the most effective low frequency component for recognition. In APIDQ, for each pixel of an input image, the intensity difference of the horizontally adjacent pixels $(d \mathrm{Ix})$ and the intensity difference of the vertically adjacent pixels ( $d$ Iy) are first calculated by using simple subtraction operations shown as formula (1). 


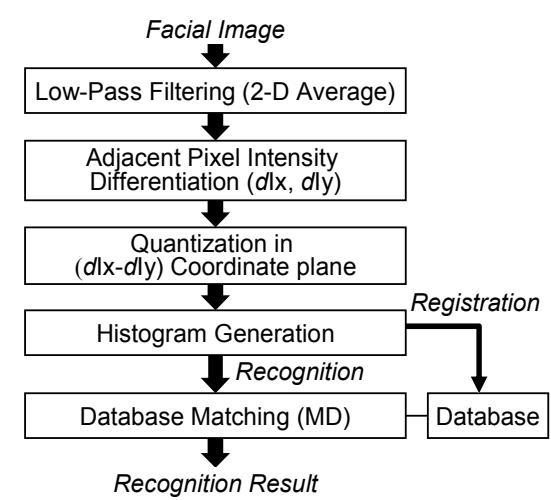

Fig. 1. Processing steps of modified APIDQ histogram method.

$$
\begin{aligned}
& d I x(i, j)=I(i+1, j)-I(i, j) \\
& d I y(i, j)=I(i, j+1)-I(i, j)
\end{aligned}
$$

A calculated $(d \mathrm{Ix}, d \mathrm{Iy})$ pair represents a single vector in the $d \mathrm{IX}-d \mathrm{Iy}$ plane. After processing all the pixels in an input image, the dots representing the vectors are distributed in the $d \mathrm{Ix}-d \mathrm{Iy}$ plane as shown in Fig. 2. The distribution of dots (density and shape) represents the features of the input image.

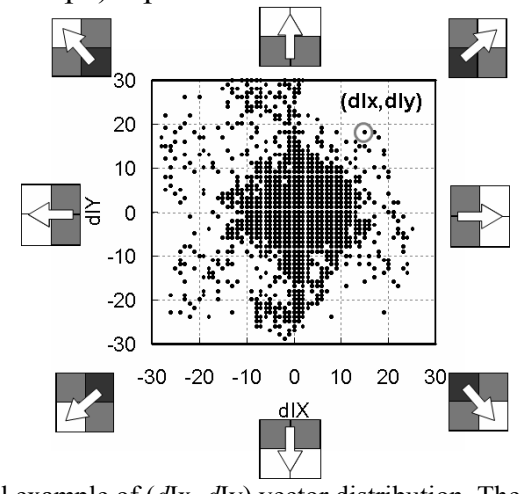

Fig. 2. Typical example of ( $d \mathrm{I} \mathrm{x}, d \mathrm{Iy})$ vector distribution. The distribution of dots (density and shape) represents the features of the input image.

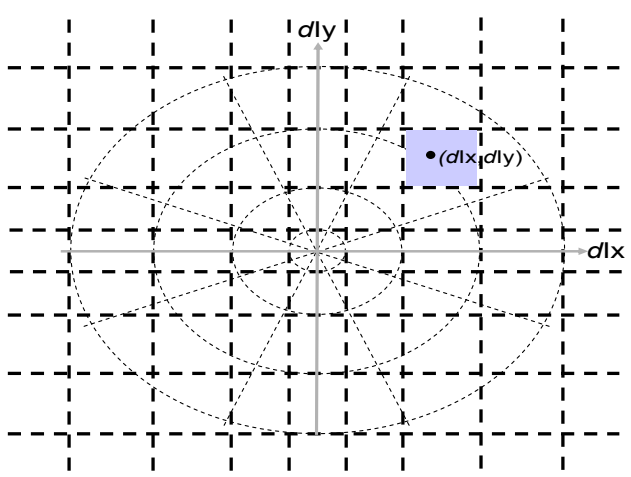

Fig. 3. Quantization in ( $d \mathrm{I} \mathrm{x}-d \mathrm{I} \mathrm{y})$ plane.

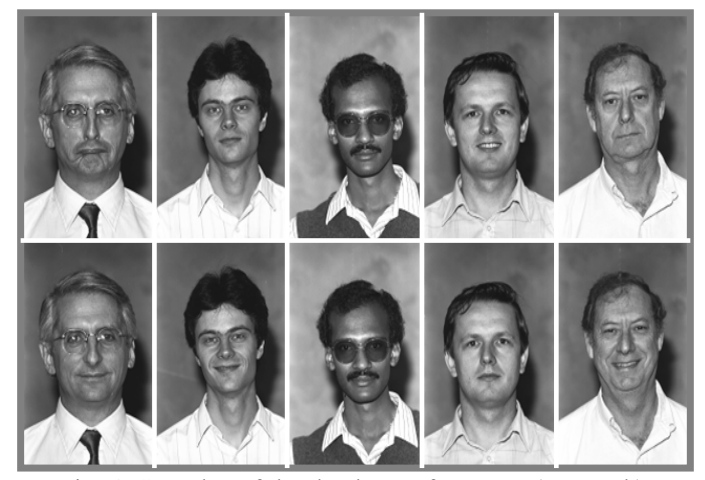

Fig. 4. Samples of the database of FERET (FB Task).

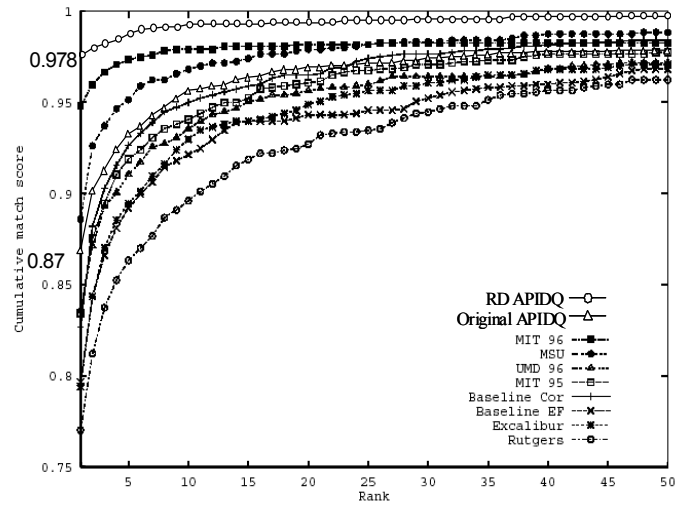

Fig. 5. Recognition results of FB task. Top1 recognition rate of $97.8 \%$ achieved is the top of the results among the all research groups

In original APIDQ [13], the coordinate system is then changed from orthogonal coordinates to polar coordinates, and each intensity variation vector is quantized in the $r-\theta$ plane as shown in Fig. 3. In this paper, we utilize the strategy that to quantize the intensity variation vectors directly in $(d \mathbf{I x}$, $d \mathrm{Iy}$ ) plane without changing to polar coordinates as shown in Fig. 3. Since $d \mathrm{Ix}-d \mathrm{Iy}$ vectors are concentrated in small- $d \mathrm{I} \mathbf{x}$, $-d$ Iy regions, non-uniform quantization steps are applied in axises. Quantization levels are set at 13 in $d \mathrm{Ix}$-axis and 13 in $d$ Iy-axis (totally 170) while the quantization steps are set to be the same as original steps of $1,2,4,7,12,20,30$. The number of vectors quantized in each quantization region is counted and a histogram is generated. This histogram becomes the feature vector of the human face. In the registration phase, this histogram is saved in a database as personal identification information. In the recognition phase, the histogram is made from an unknown input facial image and is compared to registered individual histograms and the best match is outputted as the recognition result. The Manhattan distance (MD) between histograms is used as a matching measure.

APIDQ histogram method only uses the counted histogram as the feature information to identify the people and the location information of face are unused. So we cannot know which region of facial part the matched vector point belongs to. If we could combine the histogram features and location information of the human face, the integrated features of face will be more robust and effective. Based on this idea, we developed our algorithm to a region-division (RD) APIDQ histogram method. Based on the coordinates of two eyes, which are obtained by another eye location method, inclination-revision and size-scaling processes are done to normalize the face. Then the total face area is divided into 5 regions of facial parts (forehead, eye, nose, mouth, jaw) with respective sizes, and the histograms of every region of parts are generated by APIDQ operation respectively. The histogram made from each facial region is compared with the histograms from the same facial region in the database by calculating distances $\left(\mathrm{d}_{\mathrm{i}}\right)$ between them (as shown in formula (3)). Then the integrated distances (D) are obtained by weighted averaging as shown in the following formula (2).

$$
D=\frac{\sum w_{i} d_{i}}{\sum w_{i}}, i=\text { forehead,eye, nose, mouth, jaw }
$$




$$
d_{i}=\sum_{j=1}^{33} \mid\left(\text { freq }{ }_{j}^{i n(i)}-\text { freq }_{j}^{d b(i)}\right) \mid
$$

where $w_{i}$ is weighting coefficient of the facial regions, freq ${ }_{j}^{i n(i)}$, freq $_{j}^{d b(i)}$ are the frequencies of $d \mathrm{Ix}-d$ Iy vectors that belong to a facial region of an input image and that belong to the same facial region of images registered in the database, respectively. The best match is output as recognition result by searching the minimum integrated distance.

\section{EXPERIMENTS AND DISCUSSIONS}

\section{A. Data Sets}

The publicly available face database of FERET [14] for recognition experiments, which is one of the best-known databases and consists of 14051 eight-bit grayscale images with size $256 \times 384$ of human heads with views ranging from frontal to left and right profiles. The FERET database was constructed to develop automatic face recognition capabilities that can be employed to assist security, intelligence and law enforcement personnel. We utilize FB task in our experiments. FB (fafb) holds 1195 images, which mainly assess the effect on facial expression. All the tests used a single gallery containing 1196 images. Figure 4 shows typical image samples of the FERET database. To avoid the influence of eye detection accuracy, we utilize the coordinates of eyes supplied by FERET database to implement the region-division (RD) operation in our experiments. The total face area is divided into 5 regions of facial parts (forehead, eye, nose, mouth, jaw) with sizes of $146 \times 65,146 \times 40,146 \times 30,146 \times 35,146 \times 30$, respectively.

\section{B. Experimental Results}

Fig. 5 shows the recognition results of FB obtained by using the combination of APIDQ histogram method and region-division ( $R D$ ) method to add rough location information. We get the top1 recognition rate of $87 \%$ by APIDQ histogram method directly in rectangular coordinate plane applied to the whole face area with the size of $146 \times 200$. By using RD-APIDQ histogram method with the weighting coefficient of 5 regions $1,1,0,1,1$, the top 1 recognition rate increases to $97.8 \%$. Compared with the results of other research groups who are using the same task of the FERET database, the top 1 recognition rate of $97.8 \%$ achieved is the top of the results of the whole research groups. It can be said that the modified APIDQ histogram method is a very reliable face recognition algorithm.

By applying a low pass filter, detailed facial features that degrade recognition performance, such as wrinkles, local hairstyle, image taking conditions and lapse in time, are excluded. Only the important personal facial features, such as the rough shape of facial parts, are extracted. Furthermore, APIDQ processing can effectively exclude the dc component of pixel intensity, which is simply varied by lighting conditions. By combining these two effects, the most important information for face recognition can effectively be extracted.

\section{Processing Time}

Recognition algorithm was programmed by ANSI C. To compare with the original APIDQ, the proposed method ran on the same conventional $\mathrm{PC}(3.2 \mathrm{GHz})$ as the original method. Because of the table look-up method directly in the $d \mathrm{Ix}-d \mathrm{Iy}$ domain at quantization step, the processing time for a single image in the database of FERET is $90 \mathrm{msec}$, which is composed of $50 \mathrm{msec}$ for inclination-revision and size-scaling and low-pass filtering, $15 \mathrm{msec}$ for APIDQ processing, and $25 \mathrm{msec}$ for database matching.

\section{CONCLUSIONS}

In this paper, a very fast and highly reliable modified APIDQ histogram method is proposed, which implements quantization directly in rectangular coordinate plane instead of $r$ - $\theta$ plane. Excellent face recognition performance as large as a $97.8 \%$ recognition rate has been achieved by using the publicly available database of FERET.

\section{ACKNOWLEDGMENT}

This research was partially supported by the Ministry of Education, Culture, Sports, Science and Technology of Japan, Grant-in-Aid for Young Scientists (B), No.22700089, 2010-2012.

\section{REFERENCES}

[1] K. W. Bowyer, "Face Recognition Technology and the Security versus Privacy Tradeoff," IEEE Technology and Society, pp. 9-20, 2004.

[2] R. Chellappa, C. L. Wilson, and S. Sirohey, "Human and machine recognition of faces: a survey," in Proc. IEEE, vol. 83, no. 5, pp.705-740, 1995.

[3] S. Z. Li and A. K. Jain, "Handbook of Face Recognition," Springer, New York, 2005.

[4] R. Brunelli and T. Poggio, "Face recognition: features versus templates," IEEE Trans. Pattern Analysis and Machine Intelligence, vol. 15, no. 10, pp. 1042-1052, Oct. 1993.

[5] L. Wiskott, J. M. Fellous, N. Kruger, and C. Malsburg, "Face recognition by elastic bunch graph matching," IEEE Trans. Pattern Analysis and Machine Intelligence, vol. 19, no. 10, pp. 775-780, 1997.

[6] M. Turk and A. Pentland, "Eigenfaces for recognition," Journal of Cognitive Neuroscience, vol. 3, no. 1, pp. 71-86, 1991.

[7] P. N. Belhumeur, J. P. Hespanha, and D. J. Kriegman, "Eigenface vs. Fisherfaces: Recognition using class specific linear projection," IEEE Trans. Pattern Analysis and Machine Intelligence, vol. 19, pp. 711-720, May 1997.

[8] W. Zhao, "Discriminant component analysis for face recognition," in Proc. ICPR'00, pp. 822-825, 2000.

[9] K. M. Lam and H. Yan, "An analytic-to-holistic approach for face recognition based on a single frontal view," IEEE Trans. Pattern Analysis and Machine Intelligence, vol. 20, no. 7, pp. 673-686, 1998.

[10] M. S. Bartlett, J. R. Movellan, and T. J. Sejnowski, "Face recognition by independent component analysis," IEEE Trans. on Neural Networks, vol. 13, no. 6, pp. 1450-1464, 2002.

[11] B. Moghaddam and A. Pentland, "Probabilistic visual learning for object representation," IEEE Trans. Pattern Analysis and Machine Intelligence, vol. 19, no. 7, pp. 696-710, 1997.

[12] S. G. Karungaru, M. Fukumi, and N. Akamatsu, "Face recognition in colour images using neural networks and genetic algorithms," Int. Journal of Computational Intelligence and Applications, vol. 5, no. 1, pp.55-67, 2005.

[13] K. Kotani, F. F. Lee, Q. Chen, and T. Ohmi, "Face recognition based on the adjacent pixel intensity difference quantization histogram method," 2003 Int. Symposium on Intelligent Signal Processing and Communication Systems, pp.877-880, 2003.

[14] P. J. Phillips, H. Wechsler, J. Huang, and P. Rauss. "The FERET database and evaluation procedure for face recognition algorithms," Image and Vision Computing J, vol. 16, no. 5, pp.295-306, 1998. 


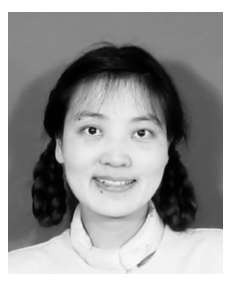

F. F. Lee received Ph.D. degree in electronic engineering from Tohoku University, Japan, in 2007. She is currently an assistant professor at New Industry Creation Hatchery Center (NICHe), Tohoku University. Her research interests include pattern recognition and multimedia processing. Dr. F. F. Lee is a member of IEEE.

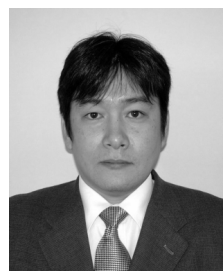

K. Kotani received the B.S., M.S. and Ph.D. degrees all in electronic engineering from Tohoku University, Japan, in 1988, 1990 and 1993, respectively. He joined the Department of Electronic Engineering, Tohoku University as a research associate in 1993. From 1997 to 1998, he belonged to the VLSI Design and Education Center (VDEC) of the University of Tokyo. He is currently an associate professor at Department of Electronic Engineering, Tohoku University. He is engaged in the research and development of high performance devices/circuits as well as intelligent electronic systems. Dr. K. Kotani is a member of IEEE and a member of the Institute of Electronics, Information and Communication Engineers of Japan

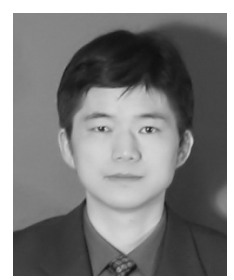

Q. Chen received $\mathrm{Ph}$. D. degree in electronic engineering from Tohoku University, Japan, in 2004 During 2004-2007, he was a COE fellow of Tohoku University 21 st century COE program. $\mathrm{He}$ is currently an associate professor at New Industry Creation Hatchery Center (NICHe), Tohoku University. His research interests include pattern recognition, computer vision, information retrieval and their applications. Dr. Q. Chen is a member of

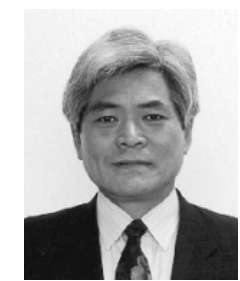

T. Ohmi served as a research associate in the Department of Electronics, Tokyo Institute of Technology, from 1966 until 1972.Then, he moved to Research Institute of Electrical Communication, Tohoku University and became an associate professor in 1976. In 1985 he became a professor at Department of Electronics, Faculty of Engineering, Tohoku University. Since 1998, he has been a professor at New Industry Creation Hatchery Center (NICHe), Tohoku University.

His research field covers whole Si-based semiconductor and flat panel display technologies in terms of material, process, device, circuit, and system technologies. He is known as an originator of "Ultralclean Technology," which introduced ultraclean and scientific way of thinking into semiconductor manufacturing industry and became indispensable technology today. Dr. T. Ohmi is a fellow of IEEE and a fellow of the Institute of Electronics, Information and Communication Engineers of Japan.

IEEE. 